\title{
Discovery of a rapidly pulsating subdwarf B star candidate in $\omega$ Centauri ${ }^{\star}$
}

\author{
S. K. Randall ${ }^{1}$, A. Calamida ${ }^{1}$, and G. Bono ${ }^{2,3}$ \\ 1 ESO, Karl-Schwarzschild-Str. 2, 85748 Garching bei München, Germany \\ e-mail: srandall@eso.org, acalamid@eso.org \\ 2 Istituto Nazionale de Astrofisica, Osservatorio Astronomico di Roma, via Frascati 33, 00040 Monte Porzio Catone, Italy \\ e-mail: bono@mporzio.astro.it \\ ${ }^{3}$ Università di Roma “Tor Vergata”, Department of Physics, via della Ricerca Scientifica 1, 00133 Rome, Italy
}

Received 31 October 2008 / Accepted 26 November 2008

\begin{abstract}
We report the discovery of the first variable extreme horizontal branch star in a globular cluster ( $\omega$ Cen). The oscillation uncovered has a period of $114 \mathrm{~s}$ and an amplitude of $32 \mathrm{mmag}$. A comparison between horizontal branch models and observed optical colours indicates an effective temperature of $31500 \pm 6300 \mathrm{~K}$ for this star, placing it within the instability strip for rapidly oscillating B subdwarfs. The time scale and amplitude of the pulsation detected are also in line with what is expected for this type of variable, thus strengthening the case for the discovery of a new subdwarf B pulsator.
\end{abstract}

Key words. stars: horizontal-branch - stars: subdwarfs - stars: oscillations - Galaxy: globular clusters: general

\section{Introduction}

Subdwarf B $(\mathrm{sdB})$ stars are hot $\left(20000 \mathrm{~K} \lesssim T_{\text {eff }} \lesssim 40000 \mathrm{~K}\right)$, compact $(5.2 \lesssim \log g \lesssim 6.2)$, evolved objects found on the extreme horizontal branch (EHB), a high temperature extension of the horizontal branch (Heber 2008). Over the last decade, a small subset $(\sim 5 \%)$ of them have been found to exhibit rapid luminosity variations with typical periods of 100-200 s and amplitudes on the mmag scale (see e.g. Fontaine et al. 2006, for a recent review). Also known as EC 14026 stars after the prototype (Kilkenny et al. 1997), the rapid pulsators are found among the hotter sdB stars in a well-defined instability strip from 29000-36000 K. Pulsation driving is believed to occur through the action of a classical $\kappa$-mechanism associated with a local overabundance of iron peak elements in the driving region, which in turn relies on radiative levitation processes (Charpinet et al. 1996, 1997). The modelling of these stars has been very successful, to the point where their non-adiabatic pulsation properties are understood not only qualitatively, but also quantitatively. Indeed, full asteroseismological analyses leading to a precise determination of the targets' fundamental parameters have now been carried out for 12 out of at least 35 known EC 14026 stars (see e.g. van Grootel et al. 2008; Charpinet et al. 2008, for recent results).

It is generally accepted that sdB stars are the progeny of red giant branch stars that underwent significant mass loss around the time of the core helium flash, leaving them with very thin hydrogen-rich envelopes. These stellar structures do not experience the asymptotic giant branch phase, instead evolving as

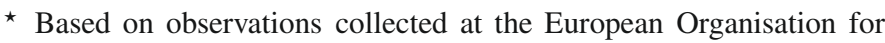
Astronomical Research in the Southern Hemisphere, Chile (proposal ID 081.D-0746) so-called AGB-Manqué stars (Greggio \& Renzini 1990), however we still lack firm constraints on the evolutionary channel(s) followed. A number of different scenarios involving e.g. a common envelope phase, stable and unstable Roche lobe overflow, or the merger of two He white dwarfs have been proposed (Han et al. 2002, 2003), but remain to be tested observationally. More recently, it has been suggested (Han 2008) that the low fraction of close binaries identified among cluster EHB stars $(\approx 4 \%$ from Moni Bidin et al. 2008) compared to the field population $(\approx 60 \%$ according to Maxted et al. 2001; $\approx 40 \%$ from Napiwotzki 2006) can be explained naturally in terms of an age effect. In the case of an older population, the white dwarf merger scenario forming a single sdB star becomes more important than common envelope evolution producing an sdB star in a close binary. It has also been suggested that cluster EHB stars might be the progeny of the "hot helium-flasher" scenario (Castellani \& Castellani 1993; D'Cruz et al. 1996; Castellani et al. 2006; Miller Bertolami et al. 2008). In this scenario, red giant stars approaching the tip of the RGB lose a significant fraction of their envelope due to a violent mass-loss event. The ensuing red giants have a higher mass than the limit for core helium ignition, and undergo a He-core flash either when approaching, or along the white dwarf cooling sequence (Brown et al. 2001). Another working hypothesis is that cluster EHB stars are the progeny of the helium-enhanced subpopulations (D'Antona et al. 2002; Lee et al. 2005; D'Antona \& Ventura 2007) recently detected in a few globular clusters (Bedin et al. 2004; Piotto 2008).

This is where asteroseismology becomes important: since the different evolutionary scenarios leave an imprint on the fundamental parameters of the resulting sdB population, contrasting the predictions from population synthesis models with the corresponding asteroseismic parameter distributions can be used to 


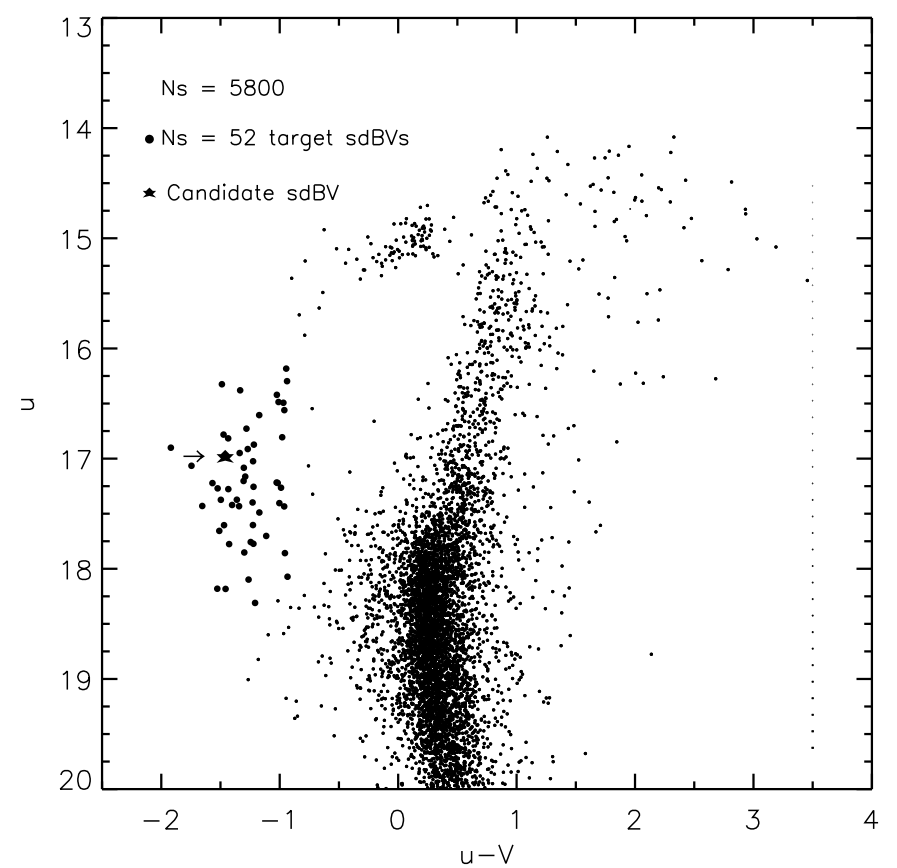

Fig. 1. CMD of $\omega$ Cen based on our SUSI $2 u$-band data combined with the WFI $V$-band data. The 52 selected subdwarf B star candidates are marked by large black points, and the position of the pulsating sdB star candidate is indicated. Error bars on the right display the mean colour and magnitude errors as a function of brightness.

distinguish between the proposed formation channels. First attempts in this direction have been made (Fontaine et al. 2008), however the number of EC 14026 stars analysed up to now is too small for quantitative conclusions. Moreover, the sample of known sdB pulsators has so far been confined to the Galactic field population. While several attempts have been made to detect variable EHB stars in globular clusters (e.g. Catelan et al. 2008; Kaluzny \& Thompson 2008; Reed et al. 2006), no convincing EC 14026 star candidates were uncovered.

Rapidly pulsating subdwarf B stars in globular clusters are of high interest in the context of an asteroseismic characterisation of the EHB population in these systems. The first necessary step in this direction is obviously the detection of pulsating subdwarf B stars in globular clusters. This is why, when given a couple of free hours at the end of a night of observations dedicated to a different programme, we decided to monitor a field in $\omega$ Cen for rapid variability among the EHB population. We present the outcome of this mini-survey in what follows.

\section{Observations}

We obtained $2 \mathrm{~h}$ of time-series photometry of a $5.5^{\prime} \times 5.5^{\prime}$ field in the southeast quadrant of $\omega$ Cen with the SUperb Seeing Imager (SUSI2) mounted on the NTT at La Silla, Chile, on 14 March 2008. A $U$-band filter was used to minimize field crowding, and $3 \times 3$ binning was chosen in order to reduce the overhead time to $16 \mathrm{~s}$. Combined with an exposure time of $20 \mathrm{~s}$, this resulted in a cycle time of $36 \mathrm{~s}$, short enough to detect the rapid oscillations expected in EC 14026 type stars.

After bias- and flatfield-correcting the images, photometry was performed using DAOPHOT/ALLSTAR and ALLFRAME (Stetson 1987, 1991, 1994). Assuming a Moffat analytical function and using the task ALLSTAR, we first estimated an analytical point-spread function (PSF) for each frame by selecting about 50 bright, isolated stars uniformly distributed over the chip. In order to obtain a global star catalogue where all images reside in the same coordinate system, we used the task DAOMATCH/DAOMASTER (Stetson 1994) to put together the 192 images obtained for the field. As a reference catalogue, we employed UBVI-band photometry of $\omega$ Cen gathered with the Wide Field Imager (WFI) on the 2.2-m ESO/MPI telescope at La Silla (Castellani et al. 2007), which completely overlaps the SUSI2 field. Finally, we performed simultaneous PSF-fitting photometry on the SUSI 2 data frames using ALLFRAME. The seeing on the individual images ranged from $0.76^{\prime \prime}$ to $1.3^{\prime \prime}$, corresponding to FWHM values between 2.9 and 5.4 pixels, while the photometric accuracy at $u \sim 18.5$ mag ranges from $\sim 0.1$ to $\sim 0.15 \mathrm{mag}$. The final star catalogue of the field monitored features 20000 stars, of which 52 were selected as subdwarf B star candidates on the basis of brightness $(16 \leq u \leq 18.5)$, colour $(-2 \leq(u-V) \leq-0.8)$, photometric accuracy $\left([\delta u, \delta V]_{\text {mean }} \leq\right.$ $0.05 \mathrm{mag})$, sharpness $(s \leq 1)$, and separation index $\left(\operatorname{sep}_{u, V} \geq\right.$ $\left.1^{1}\right)$. In Fig. 1 we show the colour magnitude diagram (CMD) obtained by cross-correlating our SUSI $u$-band data with the corresponding $V$-band measurements from the WFI catalogue. Note that the data are not calibrated in magnitude; this is however of no relevance for the present study since we are interested only in the relative brightness variation of the targets.

\section{Results}

Selecting only the 52 EHB stars indicated in Fig. 1, we computed the light curves with respect to the mean $u$-band magnitude, incorporating corrections for airmass and seeing variations by scaling the SUSI 2 magnitudes to a WFI $U$-band reference magnitude. Fourier transforms were then calculated for each light curve in the $1-15 \mathrm{mHz}$ range, appropriate for detecting the pulsations expected for the EC 14026 pulsators. We display two selected pairs of light curves and the corresponding Fourier transforms in Fig. 2. The lefthand plot is particularly interesting since it features a strong peak well above the adopted $4 \sigma$ detection threshold at a period of $114 \mathrm{~s}(8.75 \mathrm{mHz})$ and an amplitude of 30 mmag, which is compatible with EC 14026-type pulsations. The secondary peak is clearly an artefact of the data as it corresponds to the Nyquist frequency (or a period of $72 \mathrm{~s}$, twice the sampling time), and is encountered in the Fourier transform of most of the targets monitored. On the other hand, the stronger 114 s luminosity variation is unique to the star displayed, and it can be seen clearly in the phase folded light curve displayed in Fig. 4. The righthand panel of Fig. 2 features a typical EHB star from our sample that is judged constant on short time scales, with only the 72 -s $(13.89 \mathrm{mHz})$ artefact clearly visible.

The variable star uncovered has $\mathrm{J} 2000.0$ coordinates $\alpha=$ $13.27^{\mathrm{m}}$. $11.77^{\mathrm{s}}$. and $\delta=-47^{\circ} 32^{\prime} 29.0^{\prime \prime}$. A finding chart based on the combined SUSI2 image is shown in Fig. 3. It can be seen that the candidate pulsator is located close to two other stars of similar brightness, with a separation of $\sim 1.5$ times the average FWHM. In fact, space-based ACS imaging of the same region (Castellani et al. 2007) reveals a faint companion to our target that is not resolved by SUSI2 and is therefore included in the PSF computed for the target. However, the flux contribution of this companion is only $\sim 1 \%$, too small to potentially induce the $\gtrsim 3 \%$ luminosity variation detected. A verification of the photometry shows no periodic change in brightness for the

\footnotetext{
1 The separation index quantifies the degree of crowding (Stetson et al. 2003).
} 

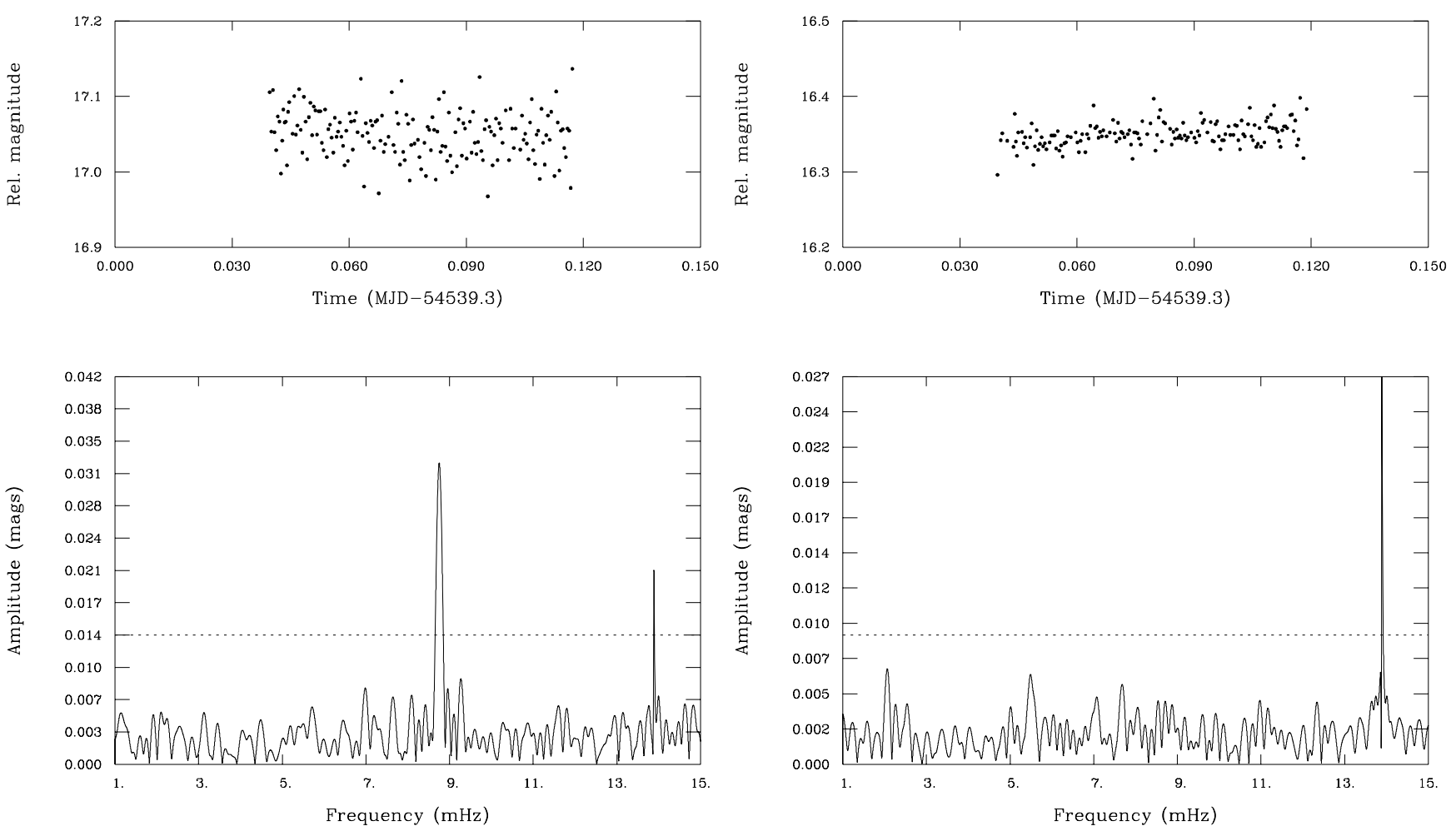

Fig. 2. Left panel: light curve (top) and Fourier transform (bottom) for the pulsating EHB star discovered in $\omega$ Cen. The relative $u$ magnitude indicated for the light curves is offset by around $+0.25 \mathrm{mag}$ with respect to the absolute magnitude from the WFI catalogue. The horizontal dashed line indicates the $4 \sigma$ detection threshold. Right panel: the same as in the left panel, but for a comparison star deemed to be constant.

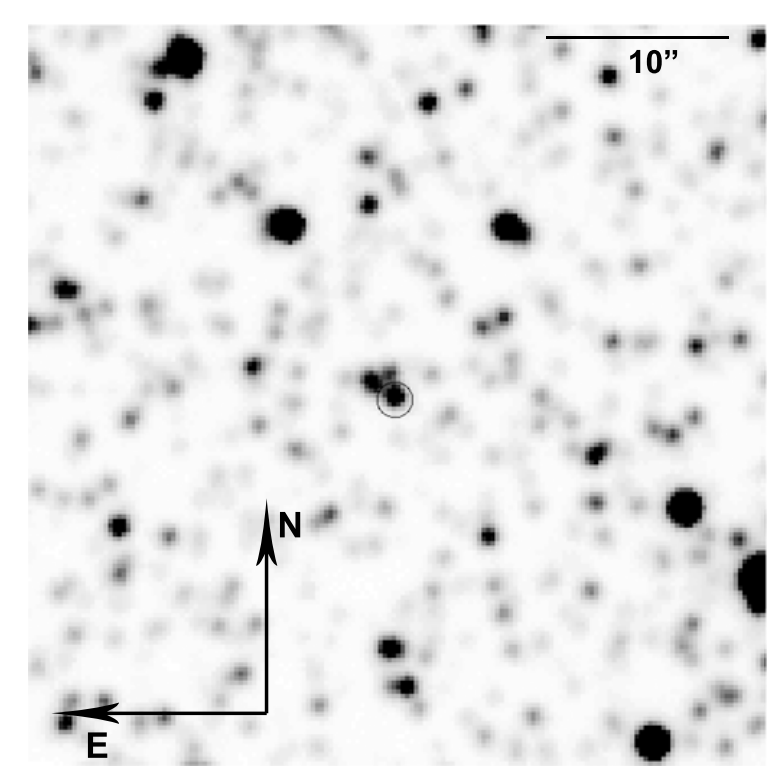

Fig. 3. Finding chart for the variable EHB star discovered in $\omega$ Cen.

stars closest to our pulsator candidate, and moreover the residuals after PSF subtraction are negligible. We therefore believe that the 114-s luminosity variation observed is inherent to the star itself rather than constituting an observational artefact.

Since the SUSI2 field overlaps with both the WFI and the ACS catalogues of $\omega$ Cen, we can determine the magnitudes and colours of the variable star in both filter systems. These are $B_{\mathrm{ACS}}=18.30, R_{\mathrm{ACS}}=18.55, H \alpha_{\mathrm{ACS}}=18.48, U_{\mathrm{WFI}}=16.81$,

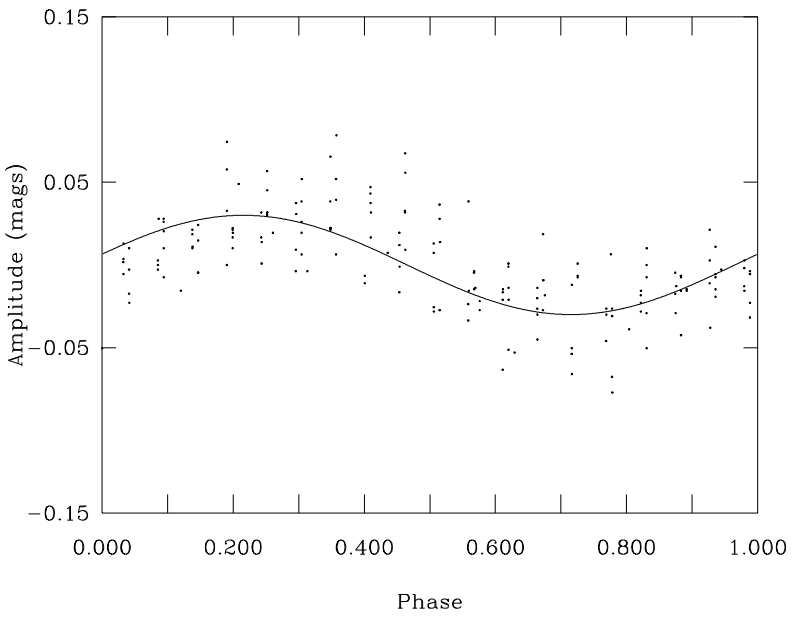

Fig. 4. Phase-folded light curve for the variable EHB star, based on the 114-s periodicity detected. The sinusoid overplotted constitutes a least squares fit to the light curve and has an amplitude of 0.03 mag and a phase of 0.22 .

$B_{\mathrm{WFI}}=18.27, V_{\mathrm{WFI}}=18.46$, and $I_{\mathrm{WFI}}=18.52$. The location of the pulsator in the CMD obtained on the basis of both the WFI and ACS data is shown in Fig. 5, and reveals this star to be a typical member of the $\omega$ Cen EHB population. We can therefore employ appropriate horizontal branch models in order to derive an estimate of the effective temperature of our target. High $\mathrm{S} / \mathrm{N}$ spectroscopy would yield more precise results, however we do not have such measurements at our disposal for the time being. In Fig. 5 we have overplotted the zero-age horizontal branch (ZAHB) and the exhaustion of central He-burning publicly 


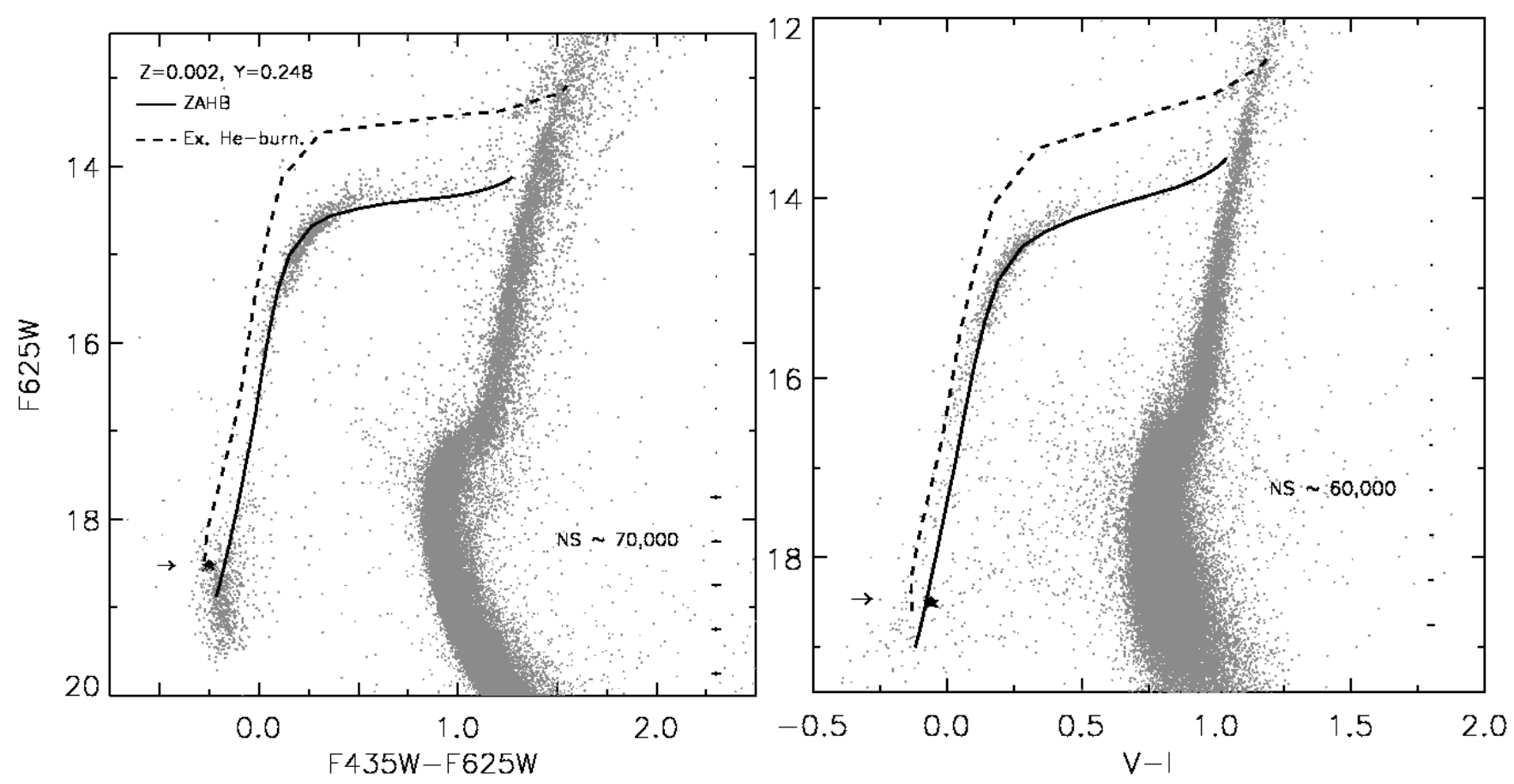

Fig. 5. F625W, F435W - F625W (left) and $I, V-I$ (right) CMDs for selected stars of the $\omega$ Cen ACS and WFI catalogues. Details concerning the selection criteria for the star catalogues used in the plot are given in Castellani et al. (2007). The error bars on the right of each plot account for colour and magnitude photometric errors, and the number of stars in each catalogue is indicated. We overplot the ZAHB and the exhaustion of central He burning for the labelled composition. The candidate sdB pulsator is marked by an arrow and asterisk in both plots.

available from the BASTI database (see, e.g. Pietrinferni et al. 2004) with an alpha enhancement $[\alpha / \mathrm{Fe}]=0.4$, helium abundance $Y=0.248$, and metallicity $Z=0.002$. We assumed a distance modulus $\mu_{0}=13.70 \pm 0.06$ (Del Principe et al. 2006) and reddening $E(B-V)=0.11 \pm 0.02$ (Calamida et al. 2005). It can be seen that in both plots the pulsator is located close to current evolutionary predictions within the theoretical and empirical errors. Given an estimated accuracy of $\sim 10 \%$ for the properties of the horizontal branch (HB) models and the slight dependency on the metallicity assumed (we averaged values for metallicities of $Z=0.002$ and $Z=0.0006$, appropriate for the metal-rich and metal-poor $\mathrm{HB}$ populations found in $\omega$ Cen) we conservatively estimate $T_{\text {eff }}=31500 \pm 6300 \mathrm{~K}$ for the variable star uncovered. This falls right into the $29000-36000 \mathrm{~K}$ instability strip for rapid sdB pulsators. The mass is estimated to be $\sim 0.5 M_{\odot}$, which is typical for a subdwarf B star. Given that the period and amplitude of the oscillation are also in line with what is expected for this type of pulsator, we believe that we have uncovered the first EC 14026 star in a globular cluster.

\section{Conclusion}

On the basis of $2 \mathrm{~h}$ of SUSI2 time-series data, as well as the availability of WFI and ACS multi-colour photometry, we discovered what appears to be the first rapidly pulsating subdwarf $\mathrm{B}$ star in a globular cluster. Both the pulsation properties and the effective temperature estimate are in line with what is observed for this type of variable in the field population; however, a longer time series of photometry and high $\mathrm{S} / \mathrm{N}$ spectroscopy are needed to verify the results presented here. It will be particularly interesting to see whether more frequencies can be uncovered, since all known sdB pulsators show multi-periodic luminosity variations. However, given the magnitude of the pulsator discovered and the shortness of the time series obtained, it is not surprising that only one periodicity was detected. Indeed, the period spectra of fast sdB pulsators are quite often dominated by one highamplitude mode, and individual frequencies can be too closely spaced to be distinguishable in just one two-hour run. The amplitude of the oscillation measured is among the highest found for any sdB pulsator (the highest amplitude pulsation is around 50 mmag for Balloon 09010001, see Oreiro et al. 2004; typical amplitudes are on the 1-5 mmag scale), and lower amplitude luminosity variations will be challenging to uncover given the faintness of the star and the crowded field. Nevertheless, we hope that the results presented here will constitute a first step towards the asteroseismology of globular cluster subdwarf B stars, and create the unique opportunity to provide quantitative constraints on their evolutionary history.

Acknowledgements. S.K.R. would like to thank the ESO La Silla staff, in particular SUSI2 instrument scientist Alessandro Ederoclite for their support. Sadly, the observations reported here were among the last ever obtained with SUSI2 since the instrument has since been decomissioned.

\section{References}

Bedin, L. R., Piotto, G., Anderson, J., et al. 2004, ApJ, 605, L125 Brown, T. M., Sweigart, A. V., Lanz, T., Landsman, W. B., \& Hubeny, I. 2001, ApJ, 562, 368

Calamida, A., Stetson, P. B., Bono, G., et al. 2005, ApJ, 634, L69

Castellani, M., \& Castellani, V. 1993, ApJ, 407, 649

Castellani, M., Castellani, V., \& Prada Moroni, P. G. 2006, A\&A, 457, 569

Castellani, V., Calamida, A., Bono, G., et al. 2007, ApJ, 663, 1021 
Catelan, M., Prieto, G. E., Zoccali, M., et al. 2008, in Hot Subdwarf Stars and Related Objects, ed. U. Heber, C. S. Jeffery, \& R. Napiwotzki, ASP Conf. Ser., 392, 347

Charpinet, S., Fontaine, G., Brassard, P., \& Dorman, B. 1996, ApJ, 471, L103

Charpinet, S., Fontaine, G., Brassard, P., et al. 1997, ApJ, 483, L123

Charpinet, S., van Grootel, V., Reese, D., et al. 2008, A\&A, 489, 377

D'Antona, F., \& Ventura, P. 2007, MNRAS, 379, 1431

D’Antona, F., Caloi, V., Montalbán, J., Ventura, P., \& Gratton, R. 2002, A\&A, 395, 69

D’Cruz, N. L., Dorman, B., Rood, R. T., \& O’Connell, R. W. 1996, ApJ, 466, 359

Del Principe, M., Piersimoni, A. M., Storm, J., et al. 2006, ApJ, 652, 362

Fontaine, G., Brassard, P., Charpinet, S., et al. 2006, in ESA SP, 624

Fontaine, G., Brassard, P., Charpinet, S., et al. 2008, in Hot Subdwarf Stars and Related Objects, ed. U. Heber, C. S. Jeffery, \& R. Napiwotzki, ASP Conf. Ser., 392, 231

Greggio, L., \& Renzini, A. 1990, ApJ, 364, 35

Han, Z. 2008, A\&A, 484, L31

Han, Z., Podsiadlowski, P., Maxted, P. F. L., Marsh, T. R., \& Ivanova, N. 2002, MNRAS, 336, 449

Han, Z., Podsiadlowski, P., Maxted, P. F. L., \& Marsh, T. R. 2003, MNRAS, 341, 669
Heber, U. 2008, in Hydrogen-Deficient Stars, ed. A. Werner, \& T. Rauch, ASP Conf. Ser., 391, 245

Kaluzny, J., \& Thompson, I. 2008, in Hot Subdwarf Stars and Related Objects, ed. U. Heber, C. S. Jeffery, \& R. Napiwotzki, ASP Conf. Ser., 392, 55

Kilkenny, D., Koen, C., O’Donoghue, D., \& Stobie, R. S. 1997, MNRAS, 285, 640

Lee, Y.-W., Joo, S.-J., Han, S.-I., et al. 2005, ApJ, 621, L57

Maxted, P. f. L., Heber, U., Marsh, T. R., \& North, R. C. 2001, MNRAS, 326, 1391

Miller Bertolami, M. M., Althaus, L. G., Unglaub, K., \& Weiss, A. 2008, ArXiv e-prints

Moni Bidin, C., Catelan, M., \& Altmann, M. 2008, A\&A, 480, L1

Napiwotzki, R. 2006, A\&A, 451, L27

Oreiro, R., Ulla, A., Pérez Hernández, F., et al. 2004, A\&A, 418, 243

Pietrinferni, A., Cassisi, S., Salaris, M., \& Castelli, F. 2004, ApJ, 612, 168

Piotto, G. 2008, Mem. Soc. Astron. Ital., 79, 334

Reed, M. D., Kilkenny, D., \& Terndrup, D. M. 2006, Baltic Astron., 15, 65

Stetson, P. B. 1987, PASP, 99, 191

Stetson, P. B. 1991, AJ, 102, 589

Stetson, P. B. 1994, PASP, 106, 250

Stetson, P. B., Bruntt, H., \& Grundahl, F. 2003, PASP, 115, 413

van Grootel, V., Charpinet, S., Fontaine, G., et al. 2008, A\&A, 488, 685 